\title{
An Unnoticed Research Method in Physics: The Special Situation Method
}

\author{
Chunqing $\mathrm{Xu}$ *, Zhongjun Chen
}

\author{
School of physics University of electronic and science technology of China, Chengdu, China \\ *Corresponding author. Email: $519634262 @ q q . c o m$
}

\begin{abstract}
It is better to teach people how to fish than to give people fishes. Physics research methods play an important role in solving practical problems. Although a physical research method is widely used, few people pay attention to it. This is the special situation method. Through the analysis of specific examples, the concept, application and practical significance of the special situation method are discussed. How to apply the special situation method and the problems needing attention in its application are also discussed.
\end{abstract}

Keywords: Physics, special situation, method

\section{INTRODUCTION}

There are many research methods in physics, and the academic circles have also made a lot of discussions on these research methods[1][2]. But there is a research method, although it is widely used, but the academia has not given due attention to this research method. This method is the special situation method. When facing a complex problem, if we can't solve it from the perspective of primary and secondary factors analysis, and can't decompose the problem into the superposition of several simple problems, we can try to find the special situation of the complex problem. In this special situation, the problem may become relatively simple, and some valuable conclusions may be obtained. This research method can be called the special situation method. Many studies have explored the use of special situation to solve mathematical problems[3][4][5]. Deng Qingyue has studied the role of "specialization" in solving physical exercises[6]. However, as an important research method of studying problems, few people pay attention to the special situations method. By combining with specific examples, this paper discusses the concept, application and practical significance of the special situation method, and analyses a series of related problems such as how to apply the special situation method and matters needing attention in its application, so as to enable this important research method to play a greater role in the process of solving practical problems.

\section{APPLICATIONS OF THE SPECIAL SITUATION METHOD}

The special situation method is a very important method to study problems, and it has a wide range of applications in physics. For example, in mechanics, the synthesis of two simple harmonic oscillations with two vibration directions vertical to each other is generally complicated, and their trajectories are chaotic and not closed. But in special situation, when the frequency of the two vibration is an integer ratio, the trajectory of the combined vibration is the famous Lissajous figure. According to the shape of the Lissajous figure, people can judge the ratio of two vibration frequencies, and use this relationship to detect the frequency of an unknown frequency signal through a signal with a known frequency. In optics, the synthesis of two light waves is generally complex, and the regularity of the synthesis wave is not obvious. But in special situation, when the frequency of two light waves is the same, the direction of vibration of the light vector is the same and the phase difference is constant, the interference phenomenon we know well will occur. In thermology, the concept of quasi-static process is a typical application of the special situation method. The intermediate state of the thermodynamic process is non-equilibrium state. The system often has no definite temperature and pressure, so it is very difficult to study the thermodynamic process. But in special situation, such as when the thermodynamic process is infinitely slow, any intermediate state experienced by the system is close to the equilibrium state, which brings great convenience for us to analyze the problem. Because the gas has definite pressure and temperature, the state parameters describing the gas satisfy the ideal gas state equation. It provides a powerful tool for the analysis of the thermodynamic process. In this process, the calculation of work, heat and entropy becomes very easy.

Not only physics, but also the special situation method has a wide range of applications in other disciplines. For example, there are too many factors affecting the stock price. Some accidental factors can make the price of a stock fluctuate enormously. In this case, people put forward the concept of efficient market, which is a typical example of the application of special situation method in finance. Effective markets are characterized by full competition in the whole market, information costs are zero, all market participants receive information at the same time, and all markets participants are rational. 
Obviously, the efficient market is a special situation of the real market. On the basis of the effective market, a series of research results which are very instructive to the analysis of the securities market have been obtained, such as the capital asset pricing model (CAPM) widely used in asset valuation, capital cost budget and resource allocation.

\section{THE SIGNIFICANCE OF RESEARCH CONCLUSION IN PRACTICE}

Some of the special situations involved in the special situation method are real, such as the examples of Lissajous figure and light interference mentioned above. In this case, the conclusions obtained by this method naturally have practical significance. Some special situations are different. In reality, these special situations does not exist strictly or rarely occurs. An example is the efficient market. The information in the market is completely symmetrical, and participants in the market can receive all kinds of information in a timely manner. These are not strictly true in the actual market. The intermediate state is almost non-equilibrium in the real thermodynamic process, and the quasi-static process is rare in the real world. What is the significance of using the special situation method in these situations? It is undeniable that the actual value of the research conclusions obtained by these special situation is indeed greatly discounted. For example, many scholars who study stocks do not necessarily make profits in the trading activities of stocks, and many conclusions using quasi-static process are not applicable in reality. But it does not mean that these conclusions are of no value at all. We also take quasi-static process as an example. If we did not study this special situation of the thermodynamic process, we would not be able to obtain the Carnot theorem, which is of great significance in thermodynamics. In many cases, if a problem is not clear about its essence, it can be analyzed in an extreme way. For example, why the equilibrium state of gas is the state with the highest entropy may be confusing, but if the problem is extreme, it is to analyze what the state with the lowest entropy of gas is like. It's easy to understand. Entropy is a sign of the degree of chaos. The lowest state of entropy is the most orderly state of gas molecules, that is, each gas molecule is arranged in an orderly manner. Obviously, the other extreme of this situation is the equilibrium state. It can be seen that the study of special situation can sometimes point out the direction of solving problems for people, just as the Carnot theorem points out a direction to improve the efficiency of heat engines.

\section{HOW TO USE SPECIAL SITUATION METHOD}

Simplification of complex problems is one of the most important research perspective in physics. This way of analyzing problem can be divided into three main types, that is, by grasping the main factors and ignoring the secondary factors, by decomposing the complex problem into the superposition of several simple problems and by studying the special situation of the complex problem. Three methods have different ideas to solve problems, each has its own advantages and complements. The specific application of these methods in practical problems requires specific analysis of specific problems. Some special situations do not exist strictly or rarely appear in reality, the actual value of the research conclusions obtained in these situations is often limited. So if the primary and secondary factors can be distinguished, the way of grasping the main factors and neglecting the secondary factors should be adopted. If complex problem can be decomposed into the superposition of several simple problems, it is advisable to use this method. If the above two methods are not feasible. we can try to find a special situation which is easy to study and draw some valuable conclusions by studying this special situation. The special situation of complex problems are often not obvious, but need to be obtained through analysis and research. Therefore, when using this method to study problems, we need to have a deep understanding of the problems studied, and then find out the special situation which are easier to study.

\section{QUESTIONS NEEDING ATTENTION}

Physics contains a series of ideas and methods that physics forefathers analyzed and solved problems for hundreds of years. It's very important to teach ideas and methods in physics class. But there is hardly any textbook of physics that gives a systematic exposition of this, and few physics teachers pay attention to this part of the content in the course of teaching. Because physics ideas and methods are contained in knowledge, in the process of learning, most students are difficult to dig it out and understand it. This requires physics teachers to integrate these valuable physical ideas and methods into physics teaching through mining and induction, so as to enhance students' independent thinking ability and problem-solving ability. The special situation method provides us with a very important research perspective. It is difficult for those who do not pay attention to ideas and methods of physics to think that they can study problems from this perspective when solving complex problems in reality. But the special situation method has not been paid attention to in physics teaching. Quasi-static process in thermodynamics is a very typical example of applying the special situation method. Few teachers can further deepen this knowledge point when teaching this part of content, and elaborate this research method to a certain extent.

In the course of teaching, students can be inspired by the way of induction, such as: in future research, students can not give up when they encounter difficult problems. Can we find out a special situation which is easier to deal with through analysis like physics forefathers and get some meaningful research results through the study of this special situation? 


\section{SUMMARY}

The special situation method obtains some valuable research conclusions by studying the special situation of complex problems. It has a wide range of applications, not only in physics, but also in other disciplines. Special situation method provides us with a very important research perspective. It is difficult for those who do not pay attention to the ideas and methods of physics to think that they can study problems from this perspective when solving complex problems in reality. Some of the special situations involved in the special situation method are real in reality, and the conclusions obtained by this method naturally have practical significance; some of them are not strict in reality or rarely appear. In this case, the practical significance of the research conclusions of the special situation method is limited, but it is not meaningless. It is better to teach people how to fish than to give them fishes. Like other methods of physics research, the special situation method should be paid attention to in physics teaching.

\section{REFERENCES}

[1] Hailan Liu, Attaching importance to the teaching of physics thought and method, physics and engineering.

19 (2009)17-20.

[2] Chunhong Zheng, Feifei Wang, Strengthen the cultivation of scientific thinking method and improve the teaching level of college physics, physics and engineering. 22(2012)52-54.

[3] Heji Yang, Mathematics learning and research of solving problem thoughts from special circumstances, Mathematics Learning and Research. 11(2013)73-75.

[4] Yaoming Zhang, The function of specialized thinking in mathematical problem solving, Mathematics Teaching Communication. 10(2000)20-21.

[5] Shaohua Zhang, Examples of the special value of "special" situation, Mathematics in Shanghai Middle School. 3(2005)19-20.

[6] Qingyue Zheng, The function of "specialization" in physical problem solving, Physics Teaching Reference in Middle School. 8(1995)28-30. 